\title{
Probióticos en diarrea aguda, asociada a antibióticos y nosocomial: evidencia en pediatría
}

\author{
Probiotics in Acute, Antibiotic-associated and Nosocomial Diarrhea: Evidence \\ in Pediatrics
}

Sebastián Posada Bustos, ${ }^{1}$ José Fernando Vera Chamorro. ${ }^{2}$

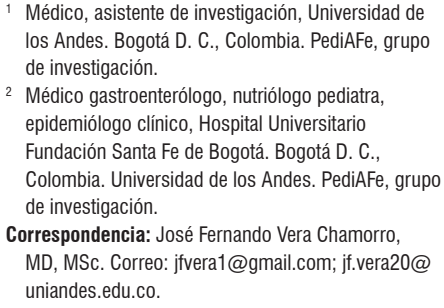
los Andes. Bogotá D. C., Colombia. PediAFe, grupo de investigación.

2 Médico gastroenterólogo, nutriólogo pediatra, epidemiólogo clínico, Hospital Universitario Fundación Santa Fe de Bogotá. Bogotá D. C. Colombia. Universidad de los Andes. PediAFe, grupo de investigación.

Correspondencia: José Fernando Vera Chamorro, MD, MSc. Correo: jfvera1@gmail.com; j.vera20@ uniandes.edu.co.

Fecha recibido: $\quad 23-05-17$

Fecha aceptado: 22-01-18

\begin{abstract}
Resumen
Introducción: los probióticos son microorganismos vivos que, administrados en cantidades adecuadas, proveen una acción benéfica en el ser humano. Existen numerosos estudios acerca de su uso en enfermedad diarreica en pediatría, por lo que se hace necesario evaluar la evidencia. Métodos: se realizó una revisión de la literatura incluyendo solo metaanálisis y revisiones sistemáticas en los últimos 10 años acerca del uso de probióticos en diarrea aguda, diarrea asociada a antibióticos y Clostridium difficile, y diarrea nosocomial. Resultados: en diarrea aguda, los metaanálisis muestran disminución de la duración en un día (intervalo de confianza [IC] 95\%; 15,9 a 33,6 horas) y disminución del riesgo de prolongación en los siguientes 4 y 7 días, con recomendaciones fuertes y evidencia moderada para Lactobacillus rhamnosus GG y Saccharomyces boulardii. En diarrea asociada a antibióticos y a C. difficile, los metaanálisis mostraron reducción del riesgo entre el $50 \%$ y $60 \%$, con recomendación fuerte para $L$. rhamnosus GG y S. boulardii con un número necesario a tratar (NNT) de 10 (IC 95\%; 7-12). En diarrea nosocomial, se encontró evidencia moderada con el uso de L. rhamnosus GG, principalmente en reducción del riesgo de gastroenteritis sintomática por rotavirus. Sin evidencia suficiente para dar recomendación para las cepas Streptococcus thermophilus y Bifidobacterium bifidum. Conclusión: solo existe evidencia con los probióticos L. rhamnosus GG y S. boulardii en reducción de la duración y disminución del riesgo de prolongación de diarrea aguda, así como reducción del riesgo entre $50 \%$ y $60 \%$ de diarrea asociada a antibióticos. Existe evidencia moderada con $L$. rhamnosus $G G$, en la reducción de riesgo de diarrea nosocomial.
\end{abstract}

Palabras clave

Probióticos, pediatría, diarrea, antibióticos, infección hospitalaria.

\section{INTRODUCCIÓN}

En 2014, la Asociación Internacional Científica para Probióticos y Prebióticos (ISAPP) ratificó y estableció la definición previamente propuesta de la Organización Mundial de la Salud (OMS) de los probióticos como "microorganismos vivos que, cuando son administrados en cantidades adecuadas, confieren un beneficio en la salud en el hospedero" (1).

Los probióticos tienen múltiples acciones fisiológicas en el cuerpo humano como son el restablecimiento de la microbiota del intestino mediante la ocupación de nichos fisiológicos para prevenir la colonización de patógenos oportunistas (2); mejoramiento de la biodisponibilidad de vitaminas, como la vitamina $\mathrm{K}, \mathrm{B}_{12}$ y piridoxina, y la síntesis de otros micronutrientes como el ácido linoleico (3); favorecimiento del tránsito intestinal para facilitar la absorción y reducir la producción de gases; y un efecto inmunomodulador mediante la acción sobre el tejido linfoide asociado con mucosas, la producción de inmunoglobulina A $(\operatorname{IgA})$ y la activación local de macrófagos; entre otros (2).

El mercado y la inversión en probióticos ha venido creciendo en grandes proporciones en los últimos años en el 


\begin{abstract}
Introduction: Probiotics are live microorganisms which, when administered in adequate amounts, provide beneficial action in humans. There are numerous studies about their use to treat diarrhea in pediatrics, so it is necessary to evaluate the evidence. Methods: We reviewed metaanalyses and systematic reviews in the last ten years about the use of probiotics to treat acute diarrhea, diarrhea associated with antibiotics and Clostridium difficile and nosocomial diarrhea. Results: Metaanalyses show that treatment of acute diarrhea with probiotics decreases duration by one day (95\% Cl: 15.9 to 33.6 hours) and reduces a risk of prolongation in the following four to seven days. They provide strong recommendations with moderate evidence for Lactobacillus rhamnosus GG and Saccharomyces boulardii. For diarrhea associated with antibiotics and Clostridium difficile, meta-analyses show risk reduction of between $50 \%$ and $60 \%$, with strong recommendations for Lactobacillus rhamnosus GG and Saccharomyces boulardii with an NNT of 10 (95\% Cl: 7 to 12). For nosocomial diarrhea, moderate evidence was found for the use of Lactobacillus rhamnosus GG, mainly in reducing the risk of symptomatic gastroenteritis due to rotavirus. Currently, sufficient evidence does not exist to give recommendation for strains $\mathrm{S}$. thermophiles and $\mathrm{B}$. bifidum. Conclusion: For reducing the duration and reducing the risk of prolongation of acute diarrhea, evidence exists only for Lactobacillus rhamnosus $G G$ and Saccharomyces boulardii. In addition, they reduce the risk of diarrhea associated with antibiotics by $50 \%$ to $60 \%$. There is moderate evidence that Lactobacillus rhamnosus GG reduces the risk of nosocomial diarrhea.
\end{abstract}

\title{
Keywords
}

Probiotics, pediatrics, diarrhea, antibiotics, hospital infections.

mundo; para 2015, el mercado de probióticos se avaluó en 33,19 billones de dólares y se proyecta que para 2020 la inversión ascienda a 46,55 billones de dólares, con una tasa anual de crecimiento del 7\% (4).

Sin embargo, no todos los productos probióticos son iguales y su uso debe depender de la calidad, seguridad, tolerabilidad, precio y eficacia, teniendo en cuenta las limitaciones económicas de nuestro sistema de salud (5). En la actualidad, estos microorganismos se han introducido al mercado como suplementos nutricionales, con estándares de aprobación más bajos que si se registraran como medicamentos, lo que garantizaría su calidad, eficacia y seguridad. (6). Esto ha llevado a la venta y promoción de múltiples productos con probióticos, de los cuales no todos tienen evidencia aceptable para su uso.

Son pocos los estudios que hablan de la calidad de los probióticos. En un estudio se evaluaron 16 productos de bifidobacterias para determinar mediante técnicas moleculares la verdadera presencia de las bacterias registradas en el empaque; se encontró que solo en uno las bifidofacterias halladas se ajustaban a los descritos en la etiqueta (7). En otros estudios se ha en encontrado que la cantidad de colonias reportadas no se pueden aislar luego de cultivarlas (8).

En los últimos años han surgido numerosos estudios acerca del uso de probióticos en enfermedad diarreica en niños, por lo que se hace necesario evaluar la evidencia disponible que aporte a la práctica clínica, su eficacia y calidad.

\section{MÉTODOS}

Se realizó una revisión de la literatura actual acerca de la evidencia de uso de probióticos en la enfermedad diarreica en pediatría, usando los términos $\mathrm{MeSH}$ : probiotics, diarrhea o gastroenteritis, antibiotic-associated diarrhea y healthcareassociated diarrhea; y se delimitó la búsqueda a metaanálisis, revisiones sistemáticas y las principales guías de práctica clínica (European Society for Paediatric Gastroenterology Hepatology and Nutrition [ESPGHAN], Sociedad Latinoamericana de Gastroenterología, Hepatología y Nutrición Pediátrica [LASPGHAN] y North American Society for Pediatric Gastroenterology, Hepatology and Nutrition [NASPGHAN]) en menores de 18 años entre 2007 y 2017. Los estudios se clasificaron en 3 categorías: diarrea aguda, diarrea asociada a antibióticos y Clostridium difficile, y diarrea nosocomial.

\section{DIARREA AGUDA}

La base del tratamiento de la enfermedad diarreica aguda es la rehidratación temprana y la realimentación precoz, así como la suplementación con zinc debido a la alta prevalencia de deficiencia en nuestro país, como lo recomienda la guía colombiana (9).

En 2010, una revisión de Cochrane analizó 63 estudios aleatorizados o cuasialeatorizados comparando probióticos contra placebo que incluyeron 8014 pacientes, de los cuales 6489 eran niños (56 estudios). En los resultados se encontró que los probióticos redujeron la duración media de la diarrea en 24,7 horas (intervalo de confianza [IC] 95\%: 15,9 a 33,6 horas), y disminuyen el riesgo de que la diarrea se prolongue por más de 4 días en un 59\%, (riesgo relativo [RR]: 0,41; IC 95\%: 0,32-0,53) (10). Los organismos más estudiados fueron Lactobacillus rhamnosus 
GG (13 estudios), Saccharomyces boulardii (10 estudios) y Enterococcus $L A B$ (lactic acid bacteria) (5 estudios) sin encontrar diferencias significativas en los desenlaces mencionados ni en las dosis utilizadas (10).

En 2013, el grupo de Applegate y colaboradores, en una revisión sistemática, evaluaron el efecto de los probióticos en niños menores de 5 años con enfermedad diarreica aguda, excluyendo lactantes y niños con uso de antibióticos; se encontraron 8 estudios que mostraban una reducción de la duración de la diarrea en un 14\% (IC 95\%: $3,8 \%-24,2 \%$ y la frecuencia de diarrea al segundo día en un 13,1\% (IC 95\%: 0,8\%-25,3\%) (11). No se encontró diferencia significativa en cuanto al tiempo de hospitalización (RR: 0,81; IC 95\%: 0,42-1,57). Dentro del subanálisis de cepas, el probiótico con evidencia de mayor consistencia fue $L$. rhamnosus $G G$, que mostró una reducción de la duración de la diarrea del 16,0\% (IC 95\%: -53,9-22,0\%) con una calidad de evidencia moderada (11).

El efecto de los probióticos depende de la especie y cepa utilizada, por lo que se han estudiado individualmente. En 2007, se publicó un metaanálisis que evaluó $S$. boulardii en diarrea aguda en niños, con 5 estudios y 619 participantes, de 2 meses hasta 12 años. En los resultados se encontró una reducción del tiempo de duración de la diarrea en 1,1 días (IC 95\%: -1,3 a -0,8 días), disminución de riesgo de persistir con diarrea a los días 3, 6 y 7 de 29\% (RR 0,71; IC 95\%: 0,56-0,90), $51 \%$ (RR 0,49; IC 95\%: 0,24-0,99) y $75 \%$ (RR: 0,25; IC 95\%: 0,08-0,83), respectivamente (12). Un estudio reportó una disminución en el tiempo de hospitalización en 1 día (IC 95\%: 1,4 a 0,62) (13).

En 2013 se publicó un metaanálisis evaluando L. rhamnosus $G G$ en gastroenteritis aguda en niños, que incluyó una población de 2963 participantes, de los cuales 11 estudios con 2444 niños demostró una reducción de la duración de la diarrea en -1,05 días (IC 95\%: -1,7 a -0,4); siendo más efectiva la dosis diaria de $\geq 10^{10}$ unidades formadoras de colonias (UFC) de L. rhamnosus GG con una disminución de la duración de la diarrea en 1,11 días (IC 95\%: -1,91 a $-0,31)$ con respecto a la dosis diaria de $<10^{10}$ UFC, con una reducción de 0,9 días (IC 95\%: -2,5 a 0,69) (14). Además, se encontró una reducción del riesgo de tener diarrea por más de 7 días en un 73\% (RR: 0,27; IC 95\%: 0,09 a 0,78). Por otra parte, se encontró que el uso de L. rhamnosus $G G$ fue efectivo en la diarrea ocasionada por rotavirus, con 3 estudios y 201 pacientes, con una reducción de 2,05 días de diarrea (IC 95\%: -2,39 a -1,71) con respecto a la de origen invasivo, en la que no mostró mejoría: 0,05 días (IC 95\%: $0,64$ a 0,74$)$; este resultado no fue estadísticamente significativo (14).

A partir de lo anterior, la ESPGHAN en 2014 estableció que debería considerarse el uso de probióticos en el manejo de la enfermedad diarreica infecciosa debido a los hallazgos en múltiples metaanálisis. De esa manera, estableció una recomendación fuerte para el uso de L. rhamnosus GG y S. boulardii; sin embargo, la evaluación de nivel de calidad de la evidencia (GRADE) fue bajo, por limitaciones en la consistencia de la evidencia y alta heterogeneidad de los metaanálisis (15). El Lactobacillus reuteri tiene una recomendación de uso débil por un nivel muy bajo de evidencia (15). Las dosis recomendadas se evidencian en la Tabla 1.

Tabla 1. Probióticos y dosis recomendadas

\begin{tabular}{|c|c|}
\hline Probiótico & Dosis \\
\hline L. rhamnosus GG & $1-2^{*} 10^{10}$ UFC/día por 5-7 días \\
\hline S. Boulardii & 250-750 mg/día por 5-7 días \\
\hline L. reuteri DSM 17938 & $1-4^{*} 10^{8}$ UCF/día por 5-7 días \\
\hline Lactobacillus acidophilus & 5 dosis de $10^{10}$ UFC $>48$ horas \\
\hline$L B$ & Máximo 9 dosis de $10^{10}$ UFC por 4,5 días \\
\hline
\end{tabular}

${ }^{*}$ Probióticos y dosis recomendadas por la ESPGHAN (15).

En 2015, un consenso latinoamericano de gastroenterología pediátrica que revisó la evidencia de alrededor de 74 artículos de recomendaciones de uso de probióticos en niños, estableció una recomendación $1 \mathrm{~A}$ para el uso de $L$. rhamnosus GG y S. boulardii en enfermedad diarreica infecciosa, y una recomendación $1 \mathrm{~B}$ para $L$. reuteri. No se especifica la dosis (16).

Por su parte, la Academia Americana de Pediatría también apoya el uso de probióticos en la enfermedad diarreica aguda infecciosa en niños sanos, específicamente L. rhamnosus $G G$ para reducir la duración de la diarrea (17).

En una revisión de guías de práctica clínica para el manejo de gastroenteritis aguda en niños a nivel mundial, se reportó que 9 de 15 guías apoyan el uso de probióticos con respecto a 10 de 15 guías que apoyan el uso de sulfato de zinc (18). En la Tabla 2 se resumen los hallazgos de metaanálisis y revisiones sistemáticas de uso de probióticos en diarrea aguda.

\section{DIARREA ASOCIADA A ANTIBIÓTICOS Y A C. DIFFICILE}

La diarrea asociada a antibióticos es una de las complicaciones más comunes del uso de antibióticos en niños dada la alteración del equilibrio biológico de la microbiota intestinal que puede llevar al crecimiento de bacterias patógenas como los bacilos de $C$. difficile (19). Se estima que tiene una incidencia del $11 \%$, los menores de 2 años son los más afectados con una incidencia hasta del $18 \%$ y los antibióticos más asociados son los betalactámicos (20).

En 2012, Videlock y colaboradores publicaron un metaanálisis acerca del uso de probióticos en diarrea asociada a antibió- 
Tabla 2. Metaanálisis y revisiones sistemáticas de uso de probióticos en diarrea aguda

\begin{tabular}{|c|c|c|c|c|}
\hline Referencia & Año & Tipo de estudio & Cepas & Resultados \\
\hline $\begin{array}{l}\text { Allen y } \\
\text { colaboradores (10) }\end{array}$ & 2010 & $\begin{array}{l}\text { Revisión } \\
\text { sistemática }\end{array}$ & $\begin{array}{l}\text { L. rhamnosus GG, S. boulardii } \\
\text { Enterococcus } L A B\end{array}$ & $\begin{array}{l}\text { Reducción de la duración de diarrea } 24,7 \text { horas } \\
\text { (IC } 95 \% \text { : } 15,9 \text { a } 33,6 \text { horas) } \\
\text { Diarrea por más de } 4 \text { días: RR: 0,41; IC 95\%: } \\
\text { 0,32-0,53 }\end{array}$ \\
\hline Applegate (11) & 2013 & $\begin{array}{l}\text { Revisión } \\
\text { sistemática }\end{array}$ & $\begin{array}{l}\text { L. bulgaricus y Streptococcus thermophilus } \\
\text { L. rhamnosus GG } \\
\text { S. boulardii } \\
\text { L. acidophilus y Bifidobacterium bifidum } \\
\text { Bacillus clausii } \\
\text { Enterococcus faecium } \\
\text { L. acidophilus } \\
\text { Lactobacillus casei, Lactobacillus plantarum, } \\
\text { Bifidobacterium infantis }\end{array}$ & $\begin{array}{l}\text { Duración de la diarrea -14,0\% (IC 95\%: - } 24,2 \text { a } \\
-3,8 \% \text { ) } \\
\text { Frecuencia de diarrea en el día 2: - } 13,1 \% \text { (IC 95\%: } \\
-25,3 \text { a }-0,8 \% \text { ) }\end{array}$ \\
\hline $\begin{array}{l}\text { Szajewska y } \\
\text { colaboradores (12) }\end{array}$ & 2007 & Metaanálisis & S. boulardii & $\begin{array}{l}\text { Reducción de la duración de la diarrea en } 1,1 \text { días } \\
\text { (IC 95\%: - } 1,3 \text { a -0,8 días) } \\
\text { Persistencia de la diarrea en: } \\
\text { Día 3: RR: 0,71; IC 95\%: 0,56-0,909 } \\
\text { Día 6: RR: 0,49; IC 95\%: 0,24-0,99 } \\
\text { Día 7: RR: 0,25; IC 95\%: 0,08-0,83 }\end{array}$ \\
\hline $\begin{array}{l}\text { Szajewska y } \\
\text { colaboradores (14) }\end{array}$ & 2013 & Metaanálisis & L. rhamnosus GG & $\begin{array}{l}\text { Reducción de la duración de la diarrea }-1,05 \text { días } \\
\text { (IC 95\%: }-1,7 \text { a }-0,4 \text { ) } \\
\text { Persistencia de diarrea a los } 7 \text { días (RR: } 0,27 \text {; IC } \\
95 \%: 0,09 \text { a } 0,78 \text { ) }\end{array}$ \\
\hline
\end{tabular}

ticos, dentro de los cuales incluyeron 10 estudios pediátricos con una población de 1246 niños. En los resultados se encontró una reducción del riesgo del $52 \%$ de desarrollar diarrea asociada a antibióticos con el uso de probióticos (RR: 0,48; IC 95\%: 0,35-0,65) independiente de la cepa usada y del tipo de antibiótico usado (21). En el subgrupo de análisis de las cepas, que incluía estudios en adultos, L. rhamnosus GG mostró una reducción del riesgo en 60\% (RR: 0,40; IC 95\%: 0,2$0,81)$ y $S$. boulardii presentó una reducción del riesgo en $56 \%$ (RR: 0,46; IC 95\%: 0,3-0,7) con menor heterogeneidad con respecto al $L$. rhamnosus $G G(21)$.

En 2015, Goldenberg y colaboradores publicaron un metaanálisis de la colaboración Cochrane, revisado en 2016 en el Journal of the American Medical Association (JAMA), que analizó 23 estudios pediátricos con una población de 3938 participantes, en el que se usaron probióticos solos o en combinación. En los resultados se encontró que la incidencia en el grupo de probióticos de diarrea asociada a antibióticos fue del 8\% (163/1992) comparado con un $19 \%(364 / 1906)$ en el grupo control, que no recibió probióticos; lo que confiere una reducción del riesgo del 54\% (RR: 0,46; IC 95\%: 0,35-0,61), con un número necesario a tratar (NNT) de 10 pacientes para prevenir un evento (IC 95\%: 7-12) (22, 23). El análisis de la evidencia
GRADE mostró una calidad de la evidencia moderada; en ningún caso se presentaron eventos adversos serios. Los probióticos con mejor evidencia fueron L. rhamnosus GG (4 estudios, $\mathrm{n}=711$ ) y $S$. boulardii (4 estudios, $\mathrm{n}=1611$ ) en dosis de 5 a 40 UFC/día $(22,24)$.

En 2015, un metaanálisis investigó la efectividad de $L$. rhamnosus $G G$ en prevención de diarrea asociada a antibióticos en adultos y niños, y se encontró una reducción del riesgo solamente en la población pediátrica del 52\% (RR: 0,48; IC 95\%: 0,26-0,89), correspondientes a 5 estudios con 455 pacientes. La calidad de la evidencia GRADE fue moderada, con un NNT de 8 pacientes para prevenir un evento (NNT: 8; IC 95\%: 6-40) (25).

En un metaanálisis que evaluaba $S$. boulardii en 6 estudios de población pediátrica para un total de 1653 pacientes encontró un riesgo del $20,9 \%$ de diarrea asociada a antibióticos en los niños con placebo, frente a un $8,8 \%$ en los niños que recibieron el probiótico, con una reducción del riesgo del 57\% (RR: 0,43; IC 95\%: 0,3-0,6) (25). Dentro del subanálisis, $S$. boulardii redujo la incidencia de diarrea asociada a $C$. difficile en un $75 \%$, en 2 estudios con 579 pacientes (RR: 0,25; IC 95\%: 0,08-0,73) (23).

Cuando se analiza específicamente el riesgo de infección por $C$. difficile, en el año 2013, una revisión sistemática de la 
colaboración Cochrane encontró que, con la administración de probióticos en el subgrupo de pacientes pediátricos, había reducción del riesgo de infección por $C$. difficile en un $60 \%$ (RR: 0,40; IC 95\%: 0,17-0,96), con un total de 3 estudios analizados, 300 pacientes con intervención y 305 controles en los que se evaluaron las cepas L. rhamnosus GG y S. boulardii (26).

En el año 2016, se publicó un metaanálisis que incluyó 4 estudios pediátricos, para un total de 938 pacientes. En los hallazgos se encontró una reducción del riesgo de infección por C. difficile en un 66\% (RR: 0,341; IC 95\%: 0,153-0,759; $\mathrm{p}=0,008)$, resultado estadísticamente significativo (27). Las cepas de L. rhamnosus GG y $S$. boulardii mostraron la mejor reducción del riesgo: $6,7 \%$ y $58,5 \%$ respectivamente; en estos últimos datos también se añade la información de pacientes adultos (27).

Por todo lo anterior, en el año 2016, la ESPGHAN revisó los datos de más de 21 estudios clínicos aleatorizados, con alrededor de 3255 pacientes que estaban incluidos dentro los metaanálisis previamente mencionados. Se encontró que el uso de probióticos redujo el riesgo de diarrea asociada a antibióticos en un 52\% (RR: 0,48; IC 95\%: 0,370,61 ) con una incidencia de $21,2 \%$ frente a $9,1 \%$ en pacientes que no recibieron probióticos (28).

La ESPGHAN reafirmó que los probióticos con mayor evidencia son L. rhamnosus $G G$ y $S$. boulardii, pero concluyó que la dosis no está claramente establecida; sin embargo, sugirió que el mayor efecto con $L$. rhamnosus $G G$ en niños se logra con dosis altas (1-2 $\times 10^{10}$ UFC) e hizo una recomendación fuerte a favor de su uso para la prevención de diarrea asociada a antibióticos, con graduación de la calidad de la evidencia moderada (28). En el caso de S. boulardii, se concluyó que no hay dosis establecida, pero no debe ser menor de $250 \mathrm{mg}$ ni mayor de $500 \mathrm{mg}$ en niños, haciendo una recomendación fuerte con una calidad de la evidencia moderada (28). Además, se hizo una recomendación adicional con calidad de evidencia baja basada en 2 estudios con 579 pacientes, quienes mostraron una reducción del $75 \%$ de riesgo de infección por $C$. difficile usando S. boulardii (RR: 0,25; IC 95\%: 0,08-0,73) $(29,30)$. Por último, se hizo mención de que el B. clausii no tiene efecto en el riesgo de diarrea asociada a antibióticos, así como tampoco la combinación de probióticos (28). Se recomienda administrar el probiótico de manera simultánea con el antibiótico y continuarlo durante la duración de dicho tratamiento.

Por su parte, el Consenso Latinoamericano emitió una recomendación $1 \mathrm{~B}$ para L. rhamnosus $G G$ y S. boulardii, para uso en diarrea asociada a antibióticos (16). En las Tablas 3 y 4 se resumen los hallazgos de metaanálisis y revisiones sistemáticas de uso de probióticos en diarrea asociada a antibióticos y a C. difficile.

\section{DIARREA NOSOCOMIAL}

Las infecciones asociadas con el cuidado de la salud o nosocomiales son aquellas que se presentan después de las

Tabla 3. Metaanálisis y revisiones sistemáticas de uso de probióticos en diarrea asociada a antibióticos

\begin{tabular}{|c|c|c|c|c|}
\hline Referencia & Año & Tipo de estudios & Cepas & Resultados \\
\hline $\begin{array}{l}\text { Videlock y } \\
\text { colaboradores (21) }\end{array}$ & 2012 & Metaanálisis & $\begin{array}{l}\text { L. rhamnosus GG } \\
\text { S. boulardii } \\
\text { L. acidophilus, B. infantis } \\
\text { Bifidobacterium longum, L. plantarum } \\
\text { B. longum, L. rhamnosus y L. plantarum }\end{array}$ & $\begin{array}{l}\text { Riesgo de diarrea: RR: 0,48; } \\
\text { IC 95\%: 0,35-0,65 }\end{array}$ \\
\hline $\begin{array}{l}\text { Goldenberg y } \\
\text { colaboradores (22) } \\
\text { Johnston B y } \\
\text { colaboradores (23) }\end{array}$ & $\begin{array}{l}2015- \\
2016\end{array}$ & Revisión sistemática & $\begin{array}{l}\text { L. acidophilus y L. casei } \\
\text { S. boulardii } \\
\text { L. casei imunitas, Lactobacillus bulgaricus y } S \text {. thermophilus } \\
\text { C. butyricum } \\
\text { L. plantarum } \\
\text { Bifidobacterium y L. acidophilus } \\
\text { L. acidophilus, L. bulgaricus, B. bifidum y S. thermophilus } \\
\text { L. acidophilus } \\
\text { Lactobacillus paracasei spp. } \\
\text { L. rhamnosus GG, L. acidophilus y Bifidobacterium }\end{array}$ & $\begin{array}{l}\text { Riesgo de diarrea: RR: 0,46; } \\
\text { IC 95\%: 0,35 a 0,61; NNT: 10; } \\
\text { IC 95\%: 7-12 }\end{array}$ \\
\hline $\begin{array}{l}\text { Szajewska y } \\
\text { colaboradores (24) }\end{array}$ & 2015 & $\begin{array}{l}\text { Revisión sistemática } \\
\text { y metaanálisis }\end{array}$ & L. rhamnosus GG & $\begin{array}{l}\text { Riesgo de diarrea: RR: 0,48; IC } \\
\text { 95\%: 0,26-0,89; NNT: 8; } \\
\text { IC 95\%: 6-40 }\end{array}$ \\
\hline Szajewska (25) & 2015 & $\begin{array}{l}\text { Revisión sistemática } \\
\text { y metaanálisis }\end{array}$ & S. boulardii & $\begin{array}{l}\text { Riesgo de diarrea: RR: 0,43; IC } \\
95 \%: 0,3-0,6\end{array}$ \\
\hline
\end{tabular}


Tabla 4. Metaanálisis y revisiones sistemáticas de uso de probióticos en diarrea asociada a C. difficile

\begin{tabular}{lcrlr}
\hline \multicolumn{1}{c}{ Referencia } & Año & Tipo de estudios & \multicolumn{1}{c}{ Cepas } & \multicolumn{1}{c}{ Resultados } \\
\hline $\begin{array}{l}\text { Goldenberg y } \\
\text { colaboradores (26) }\end{array}$ & 2013 & Revisión sistemática & $\begin{array}{l}\text { L. rhamnosus GG } \\
\text { S. boulardii }\end{array}$ & Riesgo de diarrea: RR: 0,40; IC 95\%: 0,17 a 0,96 \\
Lau y colaboradores (27) & 2016 & Revisión sistemática & $\begin{array}{l}\text { Lactobacillus GG } \\
\text { S. boulardii }\end{array}$ & Riesgo de diarrea: RR: 0,341; IC 95\%: 0,153-0,759; p: 0,008 \\
\hline
\end{tabular}

primeras 48 horas de estancia hospitalaria; y en los niños, la principal infección asociada con el cuidado de la salud es la gastroenteritis por Rotavirus (31). Un metaanálisis de 2012 mostró que la incidencia de diarrea asociada a Rotavirus es de 2,9 por cada 100 hospitalizaciones y en pico epidemiológico hasta de 8 por cada 100 hospitalizaciones (32). En este campo, el uso de probióticos parece ser efectivo en la prevención.

En 2011, se publicó un metaanálisis de 3 estudios que evaluaron el efecto de $L$. rhamnosus $G G$ en diarrea asociada al cuidado de la salud. En los resultados se encontró una reducción del riesgo de presentar diarrea tomando el probiótico en un 63\% (RR: 0,37; IC 95\%: 0,23-0,59) y de gastroenteritis sintomática por Rotavirus en un 51\% (RR: 0,49; IC 95\%: 0,28-0,86); sin embargo, no se demostró diferencia en la duración de la hospitalización (33).

En 2014, se publicó un metaanálisis que recogió datos de 6 estudios clínicos aleatorizados con 1343 pacientes, que incluyó los 3 estudios previamente mencionados acerca del uso de L. rhamnosus $G G$, confirmando los resultados previamente descritos de reducción del riesgo de presentar diarrea en un 63\% (RR: 0,37; IC 95\%: 0,23-0,59). Además, se incluyó un estudio con la combinación de B. bifidum y S. thermophilus en el que se encontró una reducción del riesgo de diarrea asociada al cuidado de la salud en un $78 \%(n=55$; RR: 0,27 ; IC 95\%: 0,08-0,87) así como de gastroenteritis sintomática por Rotavirus ( $\mathrm{n}=55$; RR: 0,27; IC 95\%: 0,08-0,87) (34). En cambio, otros 2 estudios con las cepas L. reuteri DSM 17938 y Lactobacillus delbrueckii H2B2O no mostraron ser efectivos en la reducción de diarrea nosocomial (34).
En el año 2016, se publicó un nuevo estudio que evaluó la cepa L. reuteri DSM 17938 con una población de 148 niños. No mostró diferencia en el riesgo de diarrea asociada al cuidado de la salud (RR: 1,19; IC 95\%: 0,43-3,27) (35).

Con base en lo anterior, el Consenso Latinoamericano emitió una recomendación $1 \mathrm{~B}$ para S. thermophilus, B. bifdum y $L$. rhamnosus $G G$ en la prevención de diarrea nosocomial (16). Sin embargo, en junio de 2017 la ESPGHAN publicó las recomendaciones del grupo de trabajo en probióticos en diarrea nosocomial, en las que revisó los 2 metaanálisis previamente mencionados y emitió una recomendación fuerte, con evidencia moderada a favor del uso de L. rhamnosus $G G$ (36). Debido a la evidencia de alta calidad que recomienda no usar L. reuteri DSM 17938 en diarrea nosocomial por falta de eficacia y debido a que solo se analizaron trabajos con probióticos con 2 o más estudios clínicos aleatorizados, la ESPGHAN no emitió recomendaciones por evidencia insuficiente para los probióticos S. thermophilus, B. bifidum, L. delbrueckii H2B2O y Bifidobacterium animalis subsp. lactis (BB-12 $)$ (36).

En la Tabla 5 se resumen los hallazgos de metaanálisis y revisiones sistemáticas acerca del uso de probióticos en diarrea nosocomial o asociada a al cuidado de la salud.

\section{SEGURIDAD}

Actualmente, no existen revisiones sistemáticas que analicen los efectos adversos y seguridad en la administración de probióticos. Con la evidencia actual no se ha demostrado efectos adversos serios (37); sin embargo, se han descrito casos de

Tabla 5. Metaanálisis y revisiones sistemáticas del uso de probióticos en diarrea nosocomial o asociada al cuidado de la salud

\begin{tabular}{|c|c|c|c|c|}
\hline Referencia & Año & $\begin{array}{l}\text { Tipo de } \\
\text { estudios }\end{array}$ & Cepas & Resultados \\
\hline $\begin{array}{l}\text { Szajewska y } \\
\text { colaboradores (33) }\end{array}$ & 2011 & Metaanálisis & L. rhamnosus GG & $\begin{array}{l}\text { Riesgo diarrea: RR: 0,37; IC 95\%: 0,23-0,59 } \\
\text { Riesgo de diarrea por Rotavirus: RR: 0,49; IC 95\%: 0,28-0,86 }\end{array}$ \\
\hline $\begin{array}{l}\text { Wanke y } \\
\text { colaboradores (34) }\end{array}$ & 2014 & Metaanálisis & $\begin{array}{l}\text { L. rhamnosus GG } \\
\text { B. bifidum y S. thermophilus }\end{array}$ & $\begin{array}{l}\text { Riesgo de diarrea L. rhamnosus GG: RR: 0,37; IC 95\%: 0,23-0,59 } \\
\text { Riesgo de diarrea B. bifidum y S. thermophilus: RR: 0,27; IC 95\%: } \\
0,08-0,87\end{array}$ \\
\hline
\end{tabular}


fungemia y bacteriemia secundaria a la administración de probióticos en pacientes con factores de riesgo como inmunosupresión, pacientes en unidad de cuidado intensivo (UCI) con catéter central y enfermedad grave o cardíaca estructural, por lo que no se recomienda su administración $(38,39)$.

\section{CONCLUSIÓN}

Solo existe evidencia a favor de las cepas de probióticos $L$. rhamnosus GG y $S$. boulardii en la reducción de un día de diarrea aguda así como en disminución del riesgo de prolongación de esta a los días 4 y 7 , con una recomendación fuerte de uso y una evidencia de calidad baja, por la heterogeneidad de los estudios. En cuanto a diarrea asociada a antibióticos y a C. difficile, las cepas L. rhamnosus GG y $S$. boulardii tienen evidencia de reducción del riesgo entre $50 \%-60 \%$ de diarrea con NNT de 10, evidencia de calidad moderada con recomendación fuerte de uso. Finalmente, existe evidencia a favor de L. rhamnosus $G G$ en la reducción de riesgo de diarrea nosocomial, principalmente gastroenteritis sintomática por Rotavirus; con evidencia insuficiente para los probióticos S. thermophilus y B. bifidum. El uso de las cepas Lactobacillus $G G$ y $S$. boulardii debe considerarse dentro del manejo de la enfermedad diarreica en los niños, junto con el tratamiento estándar, recordando que se debe individualizar su uso en cada paciente sopesando su costo y beneficio.

\section{Fuente de apoyo financiero}

Ninguna.

\section{Conflictos de interés}

No hay conflictos de interés en el presente artículo.

\section{REFERENCIAS}

1. Hill C, Guarner F, Reid G, et al. Expert consensus document. The International Scientific Association for Probiotics and Prebiotics consensus statement on the scope and appropriate use of the term probiotic. Nat Rev Gastroenterol Hepatol. 2014;11(8):506-14. https://doi.org/10.1038/ nrgastro.2014.66.

2. Vandenplas Y, Huys G, Daube G. Probiotics: an update. J Pediatr (Rio J). 2015;91(1):6-21. https://doi. org/10.1016/j.jped.2014.08.005.

3. Weber TK, Polanco I. Gastrointestinal microbiota and some children diseases: a review. Gastroenterol Res Pract. 2012;2012:676585.

4. MarketsandMarkets.com. Probiotics market by application (functional food \& beverages (dairy, non-dairy beverages, baked goods, meat, cereal), dietary supplements, animal feed), source (bacteria, yeast), form (dry, liquid), end user (human, animal), and region - forecast to 2022 . Marketsandmarkets.com. [internet] 2017 [acceso el 25 de abril de 2017]. Disponible en: http://www.marketsandmarkets.com/Market-Reports/probiotic-market-advancedtechnologies-and-global-market-69.html.

5. Grover S, Rashmi HM, Srivastava AK, et al. Probiotics for human health -new innovations and emerging trends. Gut Pathog. 2012;4(1):15. https://doi.org/10.1186/17574749-4-15.

6. Passariello A, Agricole P, Malfertheiner P. A critical appraisal of probiotics (as drugs or food supplements) in gastrointestinal diseases. Curr Med Res Opin. 2014;30(6):1055-64. https://doi.org/10.1185/03007995.2014.898138.

7. Lewis ZT, Shani G, Masarweh CF, et al. Validating bifidobacterial species and subspecies identity in commercial probiotic products. Pediatr Res. 2016;79(3):445-52. https:// doi.org/10.1038/pr.2015.244.

8. Goldstein EJ, Citron DM, Claros MC, et al. Bacterial counts from five over-the-counter probiotics: are you getting what you paid for? Anaerobe. 2014;25:1-4. https://doi. org/10.1016/j.anaerobe.2013.10.005.

9. Ministerio de Salud y Protección Social, Colciencias, Instituto de Evaluación Tecnológica en Salud. Guía de referencia rápida para prevención, diagnóstico y tratamiento de la enfermedad diarreica aguda en niños menores de 5 años. Bogotá D. C.: MinSalud; 2013.

10. Allen SJ, Martinez EG, Gregorio GV, et al. Probiotics for treating acute infectious diarrhoea. Cochrane Database Syst Rev. 2010;(11):CD003048. https://doi. org/10.1002/14651858.CD003048.pub3.

11. Applegate JA, Fischer Walker CL, Ambikapathi R, et al. Systematic review of probiotics for the treatment of community-acquired acute diarrhea in children. BMC Public Health. 2013;13 Suppl 3:S16.

12. Szajewska H, Skórka A, Dylag M. Meta-analysis: Saccharomyces boulardii for treating acute diarrhoea in children. Aliment Pharmacol Ther. 2007;25(3):257-64. https://doi.org/10.1111/j.1365-2036.2006.03202.x.

13. KurugölZ, Koturoğlu G. Effects of Saccharomyces boulardii in children with acute diarrhoea. Acta Paediatr. 2005;94(1):44-7. https://doi.org/10.1080/08035250410022521 - https:// doi.org/10.1111/j.1651-2227.2005.tb01786.x.

14. Szajewska H, Skórka A, Ruszczyński M, et al. Meta-analysis: Lactobacillus GG for treating acute gastroenteritis in children--updated analysis of randomised controlled trials. Aliment Pharmacol Ther. 2013;38(5):467-76. https://doi. org/10.1111/apt.12403.

15. Szajewska H, Guarino A, Hojsak I, et al. Use of probiotics for management of acute gastroenteritis: a position paper by the ESPGHAN Working Group for Probiotics and Prebiotics. J Pediatr Gastroenterol Nutr. 2014;58(4):531-9. https://doi. org/10.1097/MPG.0000000000000320.

16. Cruchet S, Furnes R, Maruy A, et al. The use of probiotics in pediatric gastroenterology: a review of the literature and recommendations by Latin-American experts. Paediatr 
Drugs. 2015;17(3):199-216. https://doi.org/10.1007/ s40272-015-0124-6.

17. Thomas DW, Greer FR; American Academy of Pediatrics Committee on Nutrition; et al. Probiotics and prebiotics in pediatrics. Pediatrics. 2010;126(6):1217-31. https://doi. org/10.1542/peds.2010-2548.

18. Lo Vecchio A, Dias JA, BerkleyJA, et al. Comparison of recommendations in clinical practice guidelines for acute gastroenteritis in children. J Pediatr Gastroenterol Nutr. 2016;63(2):22635. https://doi.org/10.1097/MPG.0000000000001133.

19. Guarino A, Guandalini S, Lo Vecchio A. Probiotics for prevention and treatment of diarrhea. J Clin Gastroenterol. 2015;49 Suppl 1:S37-45. https://doi.org/10.1097/MCG.0000000000000349.

20. Turck D, Bernet JP, Marx J, et al. Incidence and risk factors of oral antibiotic-associated diarrhea in an outpatient pediatric population. J Pediatr Gastroenterol Nutr. 2003;37(1):22-6. https://doi.org/10.1097/00005176-200307000-00004.

21. Videlock EJ, Cremonini F. Meta-analysis: probiotics in antibiotic-associated diarrhoea. Aliment Pharmacol Ther. 2012;35(12):1355-69. https://doi.org/10.1111/j.13652036.2012.05104.x.

22. Goldenberg JZ, Lytvyn L, Steurich J, et al. Probiotics for the prevention of pediatric antibiotic-associated diarrhea. Cochrane Database Syst Rev. 2015;(12):CD004827. https://doi.org/10.1002/14651858.CD004827.pub4.

23. Szajewska H, Kołodziej M. Systematic review with metaanalysis: Saccharomyces boulardii in the prevention of antibiotic-associated diarrhoea. Aliment Pharmacol Ther. 2015;42(7):793-801. https://doi.org/10.1111/apt.13344.

24. Johnston BC, Goldenberg JZ, Parkin PC. Probiotics and the prevention of antibiotic-associated diarrhea in infants and children. JAMA. 2016;316(14):1484-5. https://doi. org/10.1001/jama.2016.11838.

25. Szajewska H, Kołodziej M. Systematic review with metaanalysis: Lactobacillus rhamnosus GG in the prevention of antibiotic-associated diarrhoea in children and adults. Aliment Pharmacol Ther. 2015;42(10):1149-57. https:// doi.org/10.1111/apt.13404.

26. Goldenberg JZ, Ma SS, Saxton JD, et al. Probiotics for the prevention of Clostridium difficile-associated diarrhea in adults and children. Cochrane Database Syst Rev. 2013; (5):CD006095. https://doi.org/10.1002/14651858. CD006095.pub3.

27. Lau CS, Chamberlain RS. Probiotics are effective at preventing Clostridium difficile-associated diarrhea: a systematic review and meta-analysis. Int J Gen Med. 2016;9:27-37.

28. Szajewska H, Canani RB, Guarino A, et al. Probiotics for the prevention of antibiotic-associated diarrhea in children. $\mathrm{J}$
Pediatr Gastroenterol Nutr. 2016;62(3):495-506. https:// doi.org/10.1097/MPG.0000000000001081.

29. Kotowska M, Albrecht P, Szajewska H. Saccharomyces boulardii in the prevention of antibiotic-associated diarrhoea in children: a randomized double-blind placebo-controlled trial. Aliment Pharmacol Ther. 2005;21(5):583-90. https:// doi.org/10.1111/j.1365-2036.2005.02356.x.

30. Shan LS, Hou P, Wang ZJ, et al. Prevention and treatment of diarrhoea with Saccharomyces boulardii in children with acute lower respiratory tract infections. Benef Microbes. 2013;4(4):329-34. https://doi.org/10.3920/BM2013.0008.

31. World Health Organization. Report on the burden of endemic health care-associated infection worldwide. Géneova: WHO; 2011.

32. Bruijning-Verhagen P, Quach C, Bonten M. Nosocomial rotavirus infections: a meta-analysis. Pediatrics. 2012;129(4):e10119. https://doi.org/10.1542/peds.2011-2779.

33. Szajewska H, Wanke M, Patro B. Meta-analysis: the effects of Lactobacillus rhamnosus GG supplementation for the prevention of healthcare-associated diarrhoea in children. Aliment Pharmacol Ther. 2011;34(9):1079-87. https://doi. org/10.1111/j.1365-2036.2011.04837.x.

34. Wanke M, Szajewska H. Probiotics for preventing healthcare-associated diarrhea in children: A meta-analysis of randomized controlled trials. Pediatr Pol. 2014;89(1):8-16. https://doi.org/10.1016/j.pepo.2013.12.003.

35. Urbańska M, Gieruszczak-Białek D, Szymański H, et al. Effectiveness of Lactobacillus reuteri DSM 17938 for the prevention of nosocomial diarrhea in children: a randomized, double-blind, placebo-controlled trial. Pediatr Infect Dis J. 2016;35(2):142-5. https://doi.org/10.1097/ INF.0000000000000948.

36. Hojsak I, Szajewska H, Canani RB, et al. Probiotics for the prevention of nosocomial diarrhea in children. J Pediatr Gastroenterol Nutr. 2018;66(1):3-9. https://doi. org/10.1097/MPG.0000000000001637.

37. Hempel S, Newberry S, Ruelaz A, et al. Safety of probiotics used to reduce risk and prevent or treat disease. Evid Rep Technol Assess (Full Rep). 2011;(200):1-645.

38. Doron S, Snydman DR. Risk and safety of probiotics. Clin Infect Dis. 2015;60 Suppl 2:S129-34. https://doi. org/10.1093/cid/civ085.

39. Whelan K, Myers CE. Safety of probiotics in patients receiving nutritional support: a systematic review of case reports, randomized controlled trials, and nonrandomized trials. Am J Clin Nutr. 2010;91(3):687-703. https://doi. org/10.3945/ajcn.2009.28759. 